\title{
Tamizaje nutricional por medio de la herramienta de Ferguson en pacientes hospitalizados en un Centro Médico de la ciudad de Cali
}

\author{
Nutrition screening through the Ferguson tool in hospitalized patients in \\ a Medical Center in the city of Cali
}

\author{
Nathalia Londoño Piñeros' ${ }^{1}$ Valentina Patiño Rodríguez', Luisa Fernanda Torres', Sayda Pico Fonseca'.
}

Recibido: 25 de febrero 2018. Aceptado para publicación: 2 de marzo 2018

https://doi.org/10.35454/rncm.v1n1.073

\section{Resumen}

Introducción: la falta de aplicación de un proceso de tamizaje nutricional inicial que permita identificar en el paciente el riesgo de desnutrición en pacientes hospitalizados ha desencadenado un gran interés por parte de los profesionales encargados del cuidado clínico. La implementación de herramientas que generen un tamizaje nutricional y permita identificar de manera rápida problemas asociados a la malnutrición se hace necesario.

Objetivo: evaluar el riesgo de alteración del estado nutricional de los pacientes hospitalizados que ingresan a un centro médico de la ciudad de Cali con la herramienta de tamizaje nutricional de Ferguson.

Metodología: estudio observacional, descriptivo y transversal. La muestra la componen 236 pacientes hombres y mujeres hospitalizados (pacientes de Medicina Interna y Cirugía) entre los 18 y 59 años de edad, del Centro Médico Imbanaco. Como técnica para la recolección de datos se utilizó una herramienta tipo encuesta, durante las primeras 72 horas de hospitalización, en donde se preguntaba a los individuos datos sociodemográficos y al mismo tiempo se aplicaba el tamizaje nutricional Ferguson.

Resultados: $51,7 \%$ eran mujeres; $46,2 \%$ de los pacientes presentó un riesgo bajo de desnutrición y el 53,8 \% se encontró en riesgo medio y alto de presentar desnutrición.

Conclusiones: existe un alto porcentaje de pacientes que se encuentran con alto riesgo de desnutrición al ingreso de la hospitalización. Se hace énfasis en la importancia de realizar tamizaje nutricional con una herramienta como la Ferguson que es fiable, fácil y de bajo costo.

Palabras claves: tamizaje nutricional, herramienta de evaluación, Ferguson, desnutrición.

1 Programa de Nutrición y Dietética, Facultad de Salud y Rehabilitación Institución Universitaria Escuela Nacional del Deporte, Santiago de Cali, Colombia.

\section{Summary}

Introduction: Failure to conduct a process of initial nutritional screening which allows the risk of patient malnutrition to be identified has sparked a great interest within the professionals in charge of clinical care. Implementation of tools which render nutritional screening and allow to quickly identify issues related to malnutrition is imperative.

Objective: To assess the nutritional risk of patients admitted to a medical center in Cali by using Ferguson's nutritional screening tool.

Methodology: Cross-sectional, descriptive, observational study. The sample consists of 226 male and female patients (Internal Medicine and Surgery) aged between 18 and 59, from Imbanaco Medical Center. A survey tool was used as a method for data collection within the first 72 hours of hospital stay, in which individuals were asked for sociodemographic data and, at the same time, a Ferguson nutrition screening was performed.

Results: $51,7 \%$ of the patients were women; $46,2 \%$ of the patients had a low risk of malnutrition and $53,8 \%$ was found to be at medium and high risk of malnutrition.

Conclusions: There is a high percentage of patients with a high risk of malnutrition upon admittance to the hospital. Emphasis is placed on the importance of performing nutritional screening with a tool such as Ferguson's, which is reliable, easy to use, and low-cost.

Keywords: Nutrition screening; Assessment tool; Ferguson; Malnutrition. 


\section{INTRODUCCIÓN}

El estado nutricional de un individuo que se encuentra en el área de hospitalización es, sin lugar a duda, de gran interés para los profesionales de nutrición que se desempeñan en el área clínica, y que con los años ha empezado a cobrar gran importancia pues la nutrición hace parte del tratamiento integral durante su estancia hospitalaria ${ }^{(1)}$. El papel de un nutricionista dietista en el área clínica va más allá de atender sujetos y diseñar planes de alimentación, consiste en preocuparse por la población vulnerable que se encuentra hospitalizada a causa de las condiciones de salud y al estado patológico que presenta. Consiste también en realizar actividades tendientes a prevenir la desnutrición. Constantemente hay que buscar estrategias ya sea en la modificación gastronómica de la dieta o encontrar métodos de alimentación alternos, que permitan que los pacientes puedan cubrir su gasto energético basal con el objetivo de evitar una malnutrición en la estancia hospitalaria. El tamizaje sistemático en el marco del cuidado nutricional del paciente hospitalizado, permite identificar el riesgo nutricional de los pacientes para intervenir de manera temprana en la atención nutricional especializada ${ }^{(2,3)}$. Para la implementación del tamizaje nutricional sistemático se deben tener en cuenta los diferentes tipos de herramientas que existen para ejecutar la actividad y observar cuál se ajusta mejor a la situación, tener claro cómo se realiza la evaluación y saber qué hacer con los resultados obtenidos.

A pesar de que se han desarrollado múltiples herramientas de tamizaje no existe en la actualidad consenso sobre cuál es la mejor ni cuál herramienta utilizar ${ }^{(4,5)} \mathrm{La}$ herramienta de Ferguson o MST (Malnutrition Screening Tool) se caracteriza por su bajo costo, por ser una herramienta sencilla y rápida, y que puede ser aplicada por personal no entrenado. Además, tiene una buena capacidad para predecir morbilidad, mortalidad, y estancia hospitalaria ${ }^{(6,7)}$ Esta herramienta ha sido validada en comparación con la valoración global subjetiva ${ }^{(8,9)}$. Si el tamizaje es positivo, un profesional de la nutrición deberá realizar una evaluación nutricional completa.

Es por ello que el objetivo general del siguiente estudio es evaluar el estado nutricional de los pacientes hospitalizados que ingresan al Centro Médico Imbanaco de la ciudad Santiago de Cali con la herramienta de tamizaje nutricional de Ferguson.

\section{METODOLOGÍA}

Estudio observacional y descriptivo con enfoque de tipo corte transversal. Incluyó, por conveniencia y secuencial- mente, todos los pacientes del servicio de hospitalización del Centro Médico Imbanaco de Cali, entre febrero y junio del año 2017.

Como técnica para la recolección de datos se utilizó una herramienta tipo encuesta en donde se preguntaba a los individuos datos sociodemográficos y al mismo tiempo se aplicaba el tamizaje nutricional Ferguson. La herramienta consta de dos preguntas: 1 . ¿Ha perdido peso de manera involuntaria recientemente? $\mathrm{Si}$ ha perdido peso ¿cuántos kilogramos? 2. ¿Ha comido menos de lo normal a causa de disminución del apetito?

El estudio se ajusta a las normas éticas nacionales. La investigación se considera una investigación sin riesgo, porque solamente se recolectaron datos personales del paciente y se aplicó la encuesta sin tener contacto físico con el paciente ni ponerlo en situación de peligro.

Se tuvieron en cuenta diferentes criterios de inclusión: hombres y mujeres entre 18 y 60 años, que pertenecieran al área de hospitalización incluyendo pacientes de medicina interna y cirugía, con menos de 72 horas de ingreso, que no hubiesen tenido pérdida de algún miembro de su cuerpo y no tuviesen ningún tipo de trastorno alimenticio.

La recolección de la información se realizó a través de dos formatos: el consentimiento informado y el formato de recolección de datos sociodemográficos y que incluía la herramienta de tamizaje nutricional Ferguson también llamado 'MST' (Malnutrition Screening Tool). Este es un instrumento de cribado, diseñado para identificar adultos malnutridos y con riesgo de malnutrición (desnutrición). Está proyectado para usarse en hospitales, instituciones de atención ambulatoria y otros medios de atención sanitaria y puede ser utilizado por todos los profesionales de salud ${ }^{(5)}$.

Las variables cuantitativas fueron resumidas por medio de estadísticos de tendencia central (media) y dispersión (desviación estándar y rango). Las variables cualitativas se representaron con frecuencias absolutas y proporciones. Todos los análisis fueron realizados utilizando el software estadístico Stata $3^{\circledR}$ (StataCorp, College Station, Texas, USA).

\section{RESULTADOS}

Se incluyeron 236 pacientes hospitalizados, hombres y mujeres entre los 18 y 59 años de edad. La edad promedio de los participantes del estudio fue de 41,8 años. El $51,7 \%$ de los pacientes eran mujeres y en cuanto a la edad $67 \%$ mujeres y $69 \%$ hombres eran $>40$ años. Respecto al estrato socioeconómico, se registró desde el estrato 0 hasta el estrato 6, sin embargo, se decidió agruparlo 
entre bajo (estratos 0,1 y 2 ), medio ( 3 y 4 ) y alto ( 5 y 6). El $42,4 \%$ de los pacientes eran de estrato bajo, $35,2 \%$ de estrato medio, $21,1 \%$ de estrato alto y 3 personas no reportaron su estrato socioeconómico dado que residen fuera del país. Se observó que $89 \%$ de la población vive en zona urbana, de diferentes zonas del país, y $11 \%$ de la población vive en zona rural, principalmente en zonas del departamento del Cauca. En cuanto a la raza el 50 $\%$ de población de pacientes era de raza blanca, el $32 \%$ de raza mestiza, el $13 \%$ corresponde a la raza negra y la minoría corresponde a indígenas (Tabla 1).

Las historias clínicas de los pacientes proporcionaron información sobre si el paciente presentaba o no alguna comorbilidad, dando como resultado que el $20,8 \%$ de los pacientes eran hipertensos, el 8,1\% tenía diabetes, 3 $\%$ tenía alguna enfermedad cardiovascular y 2,5\% dislipidemias (Figura 1).

El 46,2 \% de los pacientes presentó un riesgo bajo y el $53,8 \%$ presentó un riesgo de desnutrición (Figura 2). Se agregaron el riesgo medio y alto los cuales determinan un riesgo nutricional.

Tabla 1. Datos demográficos de los pacientes

\begin{tabular}{|l|c|}
\hline \multicolumn{1}{|c|}{ Datos demográficos } & Frecuencia \\
\hline Tamaño total de la muestra (n) & 236 \\
\hline Edad (años) & \\
Media \pm SD & $41,8 \pm 11,9$ \\
Rango & $(18-60)$ \\
\hline Sexo n (\%) & \\
Femenino & $122(51,7)$ \\
Masculino & $114(48,3)$ \\
\hline Estrato Socioeconómico n (\%) & \\
Bajo & $100(42,4 \%)$ \\
Medio & $83(35,2 \%)$ \\
Alto & $50(21,1 \%)$ \\
No registra & $3(1,3 \%)$ \\
& \\
\hline Zona n (\%) & \\
Rural & $26(11,0 \%)$ \\
Urbana & $210(89,0 \%)$ \\
\hline Raza n (\%) & $12(5,1 \%)$ \\
Blanca & \\
Mestiza & \\
Negra & \\
Indígena & $(31,8 \%)$ \\
\hline Dema $\%)$ \\
\hline
\end{tabular}

SD: Desviación Estándar
Entre los pacientes que presentaron riesgo alto, el $60,3 \%$ eran pacientes mayores de 40 años y en igual proporción eran de sexo femenino (Tabla 2).

Se encontró que los pacientes de riesgo nutricional bajo son mayoritariamente de sexo masculino $(51,4 \%)$ y en el grupo de riesgo nutricional alto predomina el sexo femenino $(60,3 \%)$. En la Tabla 2 se muestra el riesgo nutricional de acuerdo con los datos demográficos y las comorbilidades.

\section{DISCUSIÓN}

Existen factores de riesgo a los que está expuesto un individuo cuando se encuentra hospitalizado, los cuales conllevan directa o indirectamente a presentar desnutrición. Algunos de estos pueden ser: la restricción en el consumo de alimentos por falta de apetito, la preparación de los alimentos, el periodo de inanición a causa de la práctica de diferentes exámenes de diagnóstico o cirugías programadas, etc. ${ }^{(6)}$. Lo anterior, podría traducirse en una estancia hospitalaria más prolongada lo que genera sobrecostos a la institución, riesgos y complicaciones a los pacientes ${ }^{(10,11)}$.

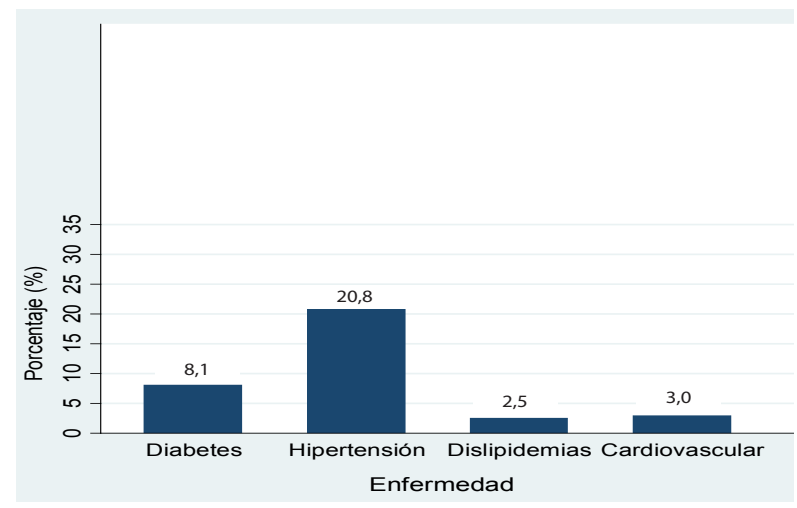

Figura 1. Porcentaje de pacientes de acuerdo con la comorbilidad

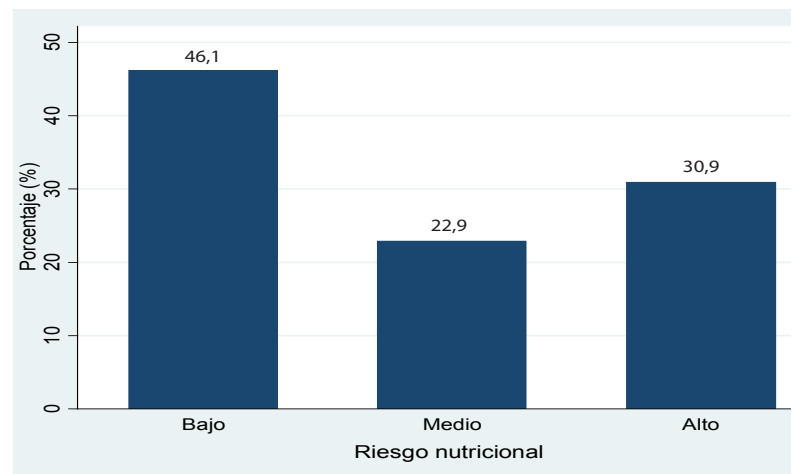

Figura 2. Riesgo nutricional de los pacientes (\%) 
Tabla 2. Riesgo nutricional de los pacientes de acuerdo con datos demográficos y comorbilidades

\begin{tabular}{|c|c|c|c|}
\hline \multirow{2}{*}{$\begin{array}{l}\text { Datos demográficos } \\
\text { y comorbilidades }\end{array}$} & \multicolumn{3}{|c|}{ Riesgo Nutricional } \\
\hline & $\begin{array}{c}\text { Bajo } \\
n=109\end{array}$ & $\begin{array}{l}\text { Medio } \\
n=54\end{array}$ & $\begin{array}{c}\text { Alto } \\
n=73\end{array}$ \\
\hline (\%) & 46,2 & 22,9 & 30,9 \\
\hline $\begin{array}{l}\text { Sexo n (\%) } \\
\text { Femenino } \\
\text { Masculino }\end{array}$ & $\begin{array}{l}53(48,6) \\
56(51,4)\end{array}$ & $\begin{array}{l}25(46,3) \\
29(53,7)\end{array}$ & $\begin{array}{l}44(60,3) \\
29(39,7)\end{array}$ \\
\hline $\begin{array}{l}\text { Raza n (\%) } \\
\text { Blanca } \\
\text { Mestiza } \\
\text { Negra } \\
\text { Indígena }\end{array}$ & $\begin{array}{c}58(53,2) \\
34(31,2) \\
12(11,0) \\
5(4,6)\end{array}$ & $\begin{array}{c}24(44,4) \\
20(37,0) \\
7(13,0) \\
3(5,6)\end{array}$ & $\begin{array}{c}36(49,3) \\
21(28,8) \\
12(16,4) \\
4(5,5)\end{array}$ \\
\hline $\begin{array}{l}\text { Zona n (\%) } \\
\text { Rural } \\
\text { Urbana }\end{array}$ & $\begin{array}{l}11(10,1) \\
98(89,9)\end{array}$ & $\begin{array}{c}8(14,9) \\
46(85,1)\end{array}$ & $\begin{array}{c}7(9,6) \\
66(90,4)\end{array}$ \\
\hline $\begin{array}{l}\text { Comorbilidades n (\%) } \\
\text { Diabetes } \\
\text { Hipertensión } \\
\text { Dislipidemias } \\
\text { Cardiovascular }\end{array}$ & $\begin{array}{c}6(5,5) \\
22(20,2) \\
4(3,7) \\
1(0,9)\end{array}$ & $\begin{array}{c}4(7,4) \\
14(26,0) \\
1(1,8) \\
3(5,5)\end{array}$ & $\begin{array}{c}9(12,3) \\
13(17,8) \\
1(1,4) \\
3(4,1)\end{array}$ \\
\hline
\end{tabular}

El tamizaje nutricional sistemático en el marco del cuidado nutricional del paciente hospitalizado, permite identificar los pacientes en riesgo y beneficiarse de la terapia nutricional ${ }^{(3)}$. La Joint Commission recomienda la detección del riesgo nutricional dentro de las 24 horas posteriores al ingreso en un hospital y a intervalos frecuentes durante la hospitalización. Sin embargo, no existe un consenso internacional sobre la mejor herramienta de tamizaje. La Asociación Colombiana de Nutrición Clínica en 2012, luego de realizar un consenso interdisciplinario y un análisis de la literatura, recomendó para Colombia la utilización de NRS 2002 o Ferguson. Se recomienda esta última por sus características de facilidad, fiabilidad y bajo costo ${ }^{(12)}$. Otras sociedades científicas recomiendan distintas herramientas (Tabla 3 ).

La prevalencia de la desnutrición se ha descrito internacionalmente entre $15 \%$ y $60 \%$ de la población hospitalaria según el tipo de paciente y la herramienta de medición que se utilice ${ }^{(13)}$. En Latinoamérica, alrededor de $50 \%$ de los pacientes adultos en los hospitales de Argentina, Brasil, Chile, Costa Rica, Cuba, República Dominicana, Ecuador, México, Panamá, Paraguay, Perú, Puerto Rico, Venezuela y Uruguay tienen algún grado de desnutrición ${ }^{(14)}$. Esto concuerda con la prevalencia de pacientes con riesgo de desnutrición obtenido en el presente estudio (53\%). Es necesario tener en cuenta que esta prevalencia es al ingreso, por tanto, es posible que le desnutrición se agrave durante la hospitalización como lo han demostrado otros estudios ${ }^{(15)}$.

Nuestros resultados también concuerdan con los obtenidos en el estudio multicéntrico observacional nutritionDay organizado en el ámbito nacional por la Asociación Colombiana de Nutrición Clínica, el cual muestra que $50 \%$ de los pacientes hospitalizados habían perdido peso y se encontraban en riesgo nutricional durante el día del estudio ${ }^{(16)}$. Además, estos son consistentes con los resultados de la aplicación de la herramienta de tamizaje nutricional a 70 pacientes en el marco del nutritionDay en la ciudad de Bucaramanga

Tabla 3. Herramientas de tamizaje nutricional hospitalaria recomendadas por las sociedades científicas internacionales. Adaptado de Cardenas D, et al ${ }^{(12)}$

\begin{tabular}{|l|l|l|l|l|}
\hline & \multicolumn{1}{|c|}{$\begin{array}{c}\text { Sociedad } \\
\text { científica }\end{array}$} & \multicolumn{1}{|c|}{ Contexto } & \multicolumn{1}{|c|}{ Población } & \multicolumn{1}{|c|}{ Objivo } \\
\hline NRS-2002, MUST & ESPEN & Hospital & $\begin{array}{l}\text { Adulto } \\
\text { Adulto mayor }\end{array}$ & $\begin{array}{l}\text { Predecir la respuesta al } \\
\text { soporte nutricional } \\
\text { ldentificar riesgo }\end{array}$ \\
\hline MNA & ESPEN & $\begin{array}{l}\text { Hospital y } \\
\text { Comunidad }\end{array}$ & Adulto mayor & + Diagnóstico de desnutrición \\
\hline MRS-2002, MUST, & ASPEN & Hospital & $\begin{array}{l}\text { Adulto } \\
\text { Adulto mayor }\end{array}$ & $\begin{array}{l}\text { Pronóstico } \\
\text { Diagnóstico }\end{array}$ \\
\hline MUST & BAPEN & $\begin{array}{l}\text { Hospital y en } \\
\text { comunidad }\end{array}$ & Adulto & $\begin{array}{l}\text { Diagnóstico de desnutrición/ } \\
\text { riesgo }\end{array}$ \\
\hline MST & Australia & Hospital & Adulto & Riesgo de desnutrición \\
\hline SNAQ & Holanda & Hospital & Adulto & Riesgo de desnutrición \\
\hline
\end{tabular}

NRS : Nutrition Risk Score; MNA: Mini Nutritional Assessment; MUST: Malnutrition Universal Screening Tool; SNAQ: Short Nutritional Assessment Questionnaire; MST o Ferguson: Malnutrition Screening Tool. 
en un hospital de alta complejidad, y población similar a la de nuestro estudio. La prevalencia de riesgo de desnutrición de dicho estudio fue similar: $52,86 \%$, (IC $95 \%$ : 40,55 \%- 64,91\%); 40,91 \% en mujeres y 58,33 $\%$ en hombres $(p=0,175)^{(17)}$.

Sin embargo, la prevalencia encontrada en nuestro estudio no es consistente con los resultados de Cruz y col. ${ }^{(18)}$. Este estudio publicado en 2017, mostró los resultados del tamizaje nutricional con la herramienta Ferguson de 295 pacientes de medicina interna en un hospital universitario en la ciudad de Bogotá. Se encontró una prevalencia de $61 \%$ de riesgo de desnutrición. Esto puede explicarse por una población más heterogénea que la de nuestro estudio y por qué se incluyeron adultos mayores. La media para la edad fue de $58,3 \mathrm{vs}$ $41,8 \pm 11,9$ en nuestro estudio. La decisión de no incluir adultos mayores en nuestro estudio se realizó buscando homogeneidad en la población. Además, porque la herramienta más indicada, que ha sido validada en esta población y que se recomienda es la Mini Nutricional Assessment (MNA) ${ }^{(19,20)}$.

Los estudios de Vega y Cruz encontraron también una asociación entre el riesgo de desnutrición, estancia hospitalaria y reingresos. Nuestro estudio no evaluó estas variables lo que se puede considerar una debilidad.

Otros estudios en Colombia han encontrado resultados similares, pero utilizando herramientas de tamizaje distintas. Por ejemplo, un estudio realizado en un hospital universitario de alta complejidad de la ciudad de Pereira en 2012, utilizando la herramienta de tamizaje nutricional NRS 2002 en 144 pacientes de todos los grupos etarios, se encontró una prevalencia de $61 \%$, siendo mayor el riesgo de padecer desnutrición en los hombres que en las mujeres. ( $69 \%$ vs $51 \% \mathrm{p}=0,036)$. Nuestro estudio mostró que el porcentaje de riesgo alto de desnutrición fue más alto en mujeres $(60,3 \% \text { vs } 39,7 \%)^{(21)}$.

Para concluir, es importante resaltar que es frecuente observar una inadecuada atención nutricional al ingreso del paciente, principalmente porque no se realizan procesos de tamizaje nutricional de manera sistemática. Al no conocer el estado nutricional del paciente que ingresa a una institución de salud, no se puede prescribir una alimentación o tratamiento adecuado para su estado de salud. Se hace énfasis en la importancia de realizar tamizaje nutricional con una herramienta como Ferguson que es fiable, fácil y de bajo costo.

\section{Agradecimientos}

Queremos agradecer a la Institución Universitaria Escuela Nacional de Deporte, al Centro Médico Imbanaco, a su personal administrativo y asistencial.

\section{Conflicto de Intereses}

Ninguno.

\section{Referencias bibliográficas}

1. Correia MI. Hospital Malnutrition in Latin America Building a Culture of Nutrition Care: The feedM.E. Global Study Group Response to "A Quick Fix for Hospital-Acquired Malnutrition?” JPEN J Parenter Enteral Nutr. 2016;40: $458-9$.

2. Mueller $\mathrm{CH}$, Compher $\mathrm{CH}$, Druyan ME, ASPEN Board of Directors. ASPEN Clinical guidelines nutrition screening, assessment, and intervention in adults. JPEN J Parenter Enteral Nutr, 2011;35:16-24.

3. Kondrup J, Allison SP, Elia M, Vellas B, Plauth M. Educational and Clinical Practice Committee, European Society of Parenteral and Enteral Nutrition. ESPEN guidelines for nutrition screening 2002. Clin Nutr. 2003;22:415-21.

4. Van Bokhorst-de van der Schueren MAE, Guaitoli PR, Jansma EP, de Vet HCW. Nutrition screening tools: Does one size fit all? A systematic review of screening tools for the hospital setting. Clin Nutr. 2014;33:39-58.

5. Elia M, Stratton RJ. An analytic appraisal of nutrition screening tools supported by original data with particular reference to age. Nutrition. 2012; 28: 477-94.

6. Skipper A, Ferguson M, Thompson K, Castellanos VH, Porcari J. Nutrition screening tools: an analysis of the evidence. JPEN J Parenter Enteral Nutr. 2012;36:292-8.

7. Lim SL, Ong KC, Chan YH, Loke WC,Ferguson M, Daniels L. Malnutrition and its impact on cost of hospitalization, length of stay, readmission and 3-year mortality. Clin Nutr. 2012;3:345-5.

8. Ferguson M, Capra S, Bauer J, Banks M. Development of a valid and reliable malnutrition screening tool for adult acute hospital patients. Nutrition. 1999;15:458-64.

9. Nursal TZ, Noyan T, Atalay BG, Köz N, Karakayali H. Simple two-part tool for screening of malnutrition. Nutrition. 2005;21:659-65.

10. Curtis LJ, Bernier P, Jeejeebhoy K,Allard J, Durksen D, Gramlich L, Laporte M, Keller HH. Costo of hospital malnutrition. Clin Nutr. 2017;36:1391-6C.

11. Asiimwe SB, Muzoora C, Wilson LA, Moore CC. Bedside measures of malnutrition and association with mortality in hospitalized adults. Clin Nutr. 2015; 34:252-6.

12. Cárdenas D, Posada C, Osorio L, Rodríguez M, Echeverri S, Cascavita M, García H. Recomendaciones de la Asociación Colombiana de Nutricion Clínica para la tamización nutricional hospitalaria en Colombia. Revista Colombiana de Metabolismo y Nutrición Clínica. 2012;3:63.

13. Norman K, Pichard C, lochs H, Pirlich M. Pronostic impact of disease-related malnutrition. Clinical Nutrition. 2008;27:5-15. 
14. Correia MI, Hegazi RA, Diaz-Pizarro Graf JI, Gomez-Morales G, Fuentes Gutiérrez C, Goldin MF, et al. Addressing DiseaseRelated Malnutrition in Healthcare: A Latin American Perspective. JPEN J Parenter Enteral Nutr. 2016;40:319-25.

15. Pinchcofsky GD, Kaminski MV Jr. Increasing malnutrition during hospitalization: documentation by a nutritional screening program. J Am Coll Nutr. 1985;4:471-9.

16. Contreras CP, Diaz G. Informe del NutritionDay 2011. Asociación Colombiana de Nutrición Clínica. Revista Colombiana de Metabolismo y Nutrición Clínica. 2012;3:105.

17. Vesga Varela AL, Gamboa Delgado EM. Risk of Malnutrition Associated with Poor Food Intake, Prolonged Hospital Stay and Readmission in a High Complexity Hospital in Colombia. Nutr Hosp. 2015;32:1308-14

18. Cruz V, Bernal L, Buitrago G, Ruiz A. Frecuencia de riesgo de desnutrición según la Escala de Tamizado para Desnutrición
(MST) en un servicio de Medicina Interna Rev Med Chile. 2017;145:449-57.

19. Drescher T, Singler K, Ulrich A, Koller M, Keller U, ChristCrain M, Kressig RW. Comparison of two malnutrition risk screening methods (MNA and NRS 2002) and their association with markers of protein malnutrition in geriatric hospitalized patients. Eur J Clin Nutr. 2010;64:887-93.

20. Bauer JM, Vogl T, Wicklein S, Trogner J, Muhlberg W, Sieber CC. Comparison of the Mini Nutritional Assessment, Subjective Global Assessment, and Nutritional Risk Screening (NRS 2002) for nutritional screening and assessment in geriatric hospital patients. Z Gerontol Geriatr. 2005;38:322-7.

21. Garcia HB, Collazos J, Acosta H, Villarreal V, Cortes C, Escarria $C$, et al. Prevalencia del riesgo nutricional en pacientes adultos a su ingreso a un hospital de tercer nivel de la ciudad de Pereira. Revista Colombiana de Metabolismo y Nutrición Clínica. 2012;3-2:105. 